\title{
RADIAL NERVE PARALYSIS IN THE NEWBORN
}

\author{
BY \\ LUCILlE MORGAN, M.B., Ch.B., D.C.H. \\ (From the Mothers' Hospital, Clapton, London)
}

Wrist drop in the newborn caused by radial nerve paralysis is a rare phenomenon, although wrist drop due to intra-uterine position of the hand, that is, acute flexion at the wrist with pressure on the flexed hand, is not very uncommon. In a survey of the literature extending over a number of years comparatively few reported cases of wrist drop due to radial nerve paralysis occurring in the newborn were found. Two babies with neonatal radial nerve paralysis born within four weeks of one another have been seen recently at the Mothers' Hospital, Clapton, and are thought worth recording. A description is also given, for comparison with the other two, of a case of wrist drop due to intrauterine pressure on the palmar flexed hand.

Case 1. Baby D had bilateral radial nerve paralysis due to antenatal trauma from a uterine contraction ring.

THE Mother. The mother was 40 years old, and had had one previous pregnancy in 1937, with normal labour.

She was admitted into hospital as an emergency because of prolonged labour. She had been in the first stage of labour for nine and a half hours, with strong frequent uterine contractions. Examination of the patient revealed a vertex presentation with no evidence of disproportion or of a pathological retraction ring (Bandl's ring). On vaginal examination the head was surrounded by an oedematous ring of cervix dilated to four fingers. In spite of good sedation and strong frequent uterine contractions every five minutes, with good relaxation between pains, labour progressed very slowly. After eight and a half hours the cervix was thought to be fully dilated, but under an anaesthetic a fine rim of cervix could be felt high up, through which the widest diameter of the head had already passed. An easy low forceps delivery was carried out under deep anaesthesia. There was some difficulty in delivering the shoulders. The cause of the delay in the first stage of labour was thought to be a contraction ring due to local tetanic uterine spasm. The reason for this diagnosis was the pressure marks on the infant, which are described below.

Condition of INFANT at BiRTH. The baby was born on Feb. 26, 1947, weighing $10 \mathrm{lb}$. $51 \mathrm{oz}$., and with white asphyxia; marked moulding of the head with caput, but no over-riding of the skull bones; cephalhaematoma of the right parietal region; bilateral wrist drop; evidence as described below of an antenatal ring of pressure encircling arms and trunk; and hypospadias (glandular). There was good recovery from white asphyxia.

The baby held its arms close to the side of the chest with the elbows acutely flexed, the forearms pronated, and the wrist, metacarpo-phalangeal, and interphalangeal joints flexed. On the lateral aspects of each arm just above the level of the elbow crease was an area of discoloured skin. The area of discoloration was roughly triangular in shape, the narrow apex of the triangle pointing forward, and measured about one and a half inches by threequarters of an inch, that of the other arm being slightly the larger. Each area consisted of a central dark blue depressed, indurated patch with one or two pin-point areas of haemorrhage surrounded by a purplish bruised margin about one-half inch wide. With the arms at the side of the chest this band and the two pressure marks on the arms were at exactly the same level. The baby was unable to dorsiflex the wrists, supinate the forearms, or extend the fingers. The creases at the wrists, showing as a band of smooth pale pink skin, and the position (close to the chest) in which the arms were held suggested that antenatally the elbows had been acutely flexed and each hand had been tucked in a position of flexion into the axilla of the same side. There were no pressure marks on the forearms, hence the forearms must have been sufficiently extended at the elbow at the time the contraction ring developed to escape the direct pressure effect of the ring.

Treatment. The forearms were splinted with the wrists in dorsiflexion and the fingers were passively extended daily.

Progress. Three days after delivery the constriction mark across the chest was no longer visible, although there was no noticeable improvement in the marks on the arms. Within a week from birth the baby was extending the forearms and waving the splints around. Extension of the fingers slowly improved, although there was as yet no active attempts at dorsiflexion of the wrists. By the eighth day the baby was able slightly to dorsiflex the wrists, the left a little more than the right. The amount of induration in the pressure areas on the arms increased, and reddish patches of pseudosclerema appeared in the skin and subcutaneous tissue of the neck and the back. The 
bruised-like zone of pseudosclerema felt to the examiner not unlike the indurated areas in the arms. When the baby was discharged at 19 days old there was nearly full power of dorsifexion at the wrists but full active extension of the fingers was not possible. At three months old, when seen again, the baby looked a healthy well-grown infant, the pseudosclerema had disappeared, and there was full range of movement in both upper limbs. There still remained in each arm a linear scar involving the skin and subcutaneous tissue, but the discoloration and induration had disappeared.

Case 2. Baby B had right radial nerve paralysis resulting from an obstetrical fracture of the humerus.

The Mother. The mother was 24 years old, a primigravida. There was forceps delivery for primary uterine inertia continuing into the second stage.

Condition of INfant at Birth. The child was born on March 22, 1947, and weighed $8 \mathrm{lb} .4 \mathrm{oz}$. The general condition was good, but there was simple transverse fracture of the humerus just above the middle of the shaft, with no obvious deformity. This was later confirmed by radiograph. All movements of the fingers and wrist joint, and the movements of flexion and extension of the elbow joint was present at birth. While the baby was undisturbed the upper arm lay flaccid by the side of the chest and there was no attempt on the part of the baby to move it.

Treamment: The upper arm was splinted with three straight splints, the elbow being flexed across the chest.

Progress. A week later the characteristic posture of wrist drop had developed, with no active dorsiflexion of the wrists or extension of the metacarpo- and interphalangeal joints. The muscular tone of the forearm was diminished and there was some ulnar deviation. Owing to the age of the infant it was impossible to estimate the changes in skin sensitivity. There did not appear to have been any pressure in the axilla from the splints. A diagnosis of radial paralysis was made and the forearm was splinted with the wrist dorsifiexed.

Within three days the baby was able slightly to dorsifex the wrist and extend the fingers. Full movement of the wrist and the fingers rapidly returned, and the forearm splint was removed seventeen days after delivery. The fracture of the humerus healed completely within one month from birth, and when discharged the infant had no evidence of radial paralysis and only a small amount of callus at the site of the fracture.

Case 3. Baby $\mathbf{H}$ had left wrist drop due to intra-uterine palmar flexion.

THE MOTHER. The mother was a primigravida 37 years old. She had forceps delivery for primary uterine inertia, continuing into the second stage.

Condmion of Infant at Birth. The baby was born on March 23, 1947, weighing $8 \mathrm{lb} .4 \mathrm{oz}$. The general condition was good. The left arm was held closely to the side of the chest with the elbow flexed. The forearm was pronated, the wrist palmar flexed, and the fingers clenched into the palm. The clenched hand fitted snugly into a depression below the left ear. There was a small area of red skin on the left arm near the insertion of the deltoid, but this was accounted for by the fact that the skin creased at this spot when the hand was held in the hollow below the left ear. The baby was unable to extend the hand at the wrist joint.

Treatment. A splint was applied to the left forearm with the hand slightly dorsiflexed.

Progress. At one week old the baby could fully extend the fingers at all joints with the wrist flexed. The hand still assumed the position of wrist drop, but the wrist extensors were. beginning to recover. When the infant was next seen at the out-patient clinic, shortly after discharge from the ward, there was full range of movement.

\section{Discruscion}

The wrist drop in case 3 was clearly brought about by intra-uterine position, and intra-uterine position was considered as a possible cause of the wrist drop in case 1. However, in this case there was evidence of trauma of the soft tissues from pressure in an area where the radial nerve would be likely to be involved, and therefore it is reasonable to conclude that the trauma caused the paralysis.

Moreover, at least four other cases have been described closely resembling case 1 , in each of which there was evidence of a band of pressure surrounding the trunk and arm and causing local trauma and radial nerve paralysis. The first was described by Brun in 1933 (quoted by Hauch and Ottsen, 1939). The baby had a dark, elongated transverse pressure mark on the outer side of the left arm, and on the same level a similar transverse pressure mark on the trunk. The mother was in labour four days, and the author attributed the baby's left radial nerve paralysis to the pressure of a "Bandl's ring " before birth.

It appears that the German authors apply the term " Bandl's ring " to the contraction ring, but in this country the name " Bandl's ring" is restricted to the retraction ring. The retraction ring (Bandl's ring of English textbooks) is a groove at the junction of the upper contracting and the lower passive uterine segments. It is present in every labour but does not normally rise above the symphysis. In obstructed labour, however, it becomes evident, running across the uterus. A contraction ring is a local spasm of the circular muscle of the uterus. It usually occurs in the lower uterine segment during the second stage of labour, but may occur anywhere in the uterus during any stage. The contraction 
ring exerts great pressure and no progress occurs in the labour though pains continue.

Hauch and Ottsen in 1939 described another infant with left radial paralysis, also with a linear transverse pressure zone across the left arm and across the back on the same level. As in our case, there was deep infiltration of the tissue of the arm in the zone of pressure. The degree of trauma of the trunk was greater than in our case, and a biopsy, when the infant was one month old, of tissue from the zone of pressure showed inflammatory changes in the subcutaneous connective tissue and traumatic fatty necrosis. The authors consider that this may indicate the existence of previous "trophoneurotic " changes in the tissues predisposing to the pressure effect, which is interesting in view of the fact that pseudo-sclerema (localized subcutaneous fat necrosis) developed in our patient after birth. The mother had a long labour. Hauch and Ottsen considered there was no evidence to prove the existence of a "Bandl's ring" in this case, presumably using this term in the sense of a contraction ring, and attributed the long labour to a rigid cervix, but left undecided the origin of this transverse zone of pressure, as they were not able to suggest any other likely explanation.

Kehrer in 1918 gave an account of a new-born baby with bilateral radial nerve paralysis and pressure marks just below the middle of both upper arms. The marks on the outer side of each arm were thought to be due to pressure from the promontory and symphysis in a flat rachitic pelvis. It is not clear whether he considered the possibility of a uterine contraction ring, and one is tempted to suppose that these three cases may have been due, like ours, to such a ring of constriction.

A fourth case of bilateral radial nerve paralysis found by Hauch and Ottsen in their hospital records was born to a mother with a long labour and again a 'Bandl's ring.' Hauch and Ottsen in 1939 were able to find twenty-five cases of neonatal radial nerve paralysis in the literature, found six more in their hospital records, and added two more cases of their own, making thirty-two in all. All these were apparently due to trauma of the peripheral part of the radial nerve. It is surprising that only two of these were due to involvement of the nerve at the site of a fracture and it seems probable that this etiology is more common than the figures would suggest.

The prognosis of neonatal radial nerve paralysis is good.

\section{Summary}

Two cases of radial nerve paralysis in the newborn are presented, one due to pressure from a uterine contraction ring, the other due to involvement of the radial nerve at the site of a fracture.

I wish to thank Dr. H. M. M. Mackay for her permission to publish these cases, and for her encouragement and helpful advice.

\section{REFERENCES}

Hauch, E. and Ottsen, M. (1939). Acta Obstet. Gymaec. Scand., 19, 345.

Kehrer, E. (1918). Zbl. Gynäk, 42, 563.

(1919). Ibid., 43, 338. 